\title{
New Variables for Classical and Quantum (Super)-Gravity in all Dimensions
}

\author{
Norbert Bodendorfer, Thomas Thiemann and Andreas Thurn \\ Institute for Theoretical Physics III, FAU Erlangen - Nürnberg, Staudtstr. 7, 91058 Erlangen, \\ Germany \\ E-mail: norbert.bodendorferegravity.fau.de, \\ thomas.thiemannegravity.fau.de, andreas.thurnegravity.fau.de
}

\begin{abstract}
Supergravity was originally introduced in the hope of finding a theory of gravity without the shortcoming of perturbative non-renormalisability. Although this goal does not seem to have been reached today (with the possible exception of $d=4, N=8$ Supergravity [1]), Superstring theories are argued to reproduce $10 d$ Supergravities as their low energy limits and thus provide a UV-completion of those theories. Loop Quantum Gravity (LQG) on the other hand is a manifestly background independent and non-perturbative approach to the quantisation of General Relativity, however presently limited to four spacetime dimensions. In order to make contact between these two approaches to quantum gravity, we extend the techniques of LQG to higher dimensions and new matter fields appearing in Supergravity theories. The immediate applications of our framework for further research are comparisons between symmetry reduced sectors of those two theories, namely cosmology and black holes.
\end{abstract}

PACS: $04.65 .+e, 04.60 . P p, 04.20 . F y, 04.50 .-h$

3rd Quantum Gravity and Quantum Geometry School,

February 28 - March 13, 2011

Zakopane, Poland 
The programme of Loop Quantum Gravity was initiated by Ashtekar's discovery of his new variables [2] in 1986. During the next 10 years, the technical foundations of the programme were developed and it transpired that LQG naturally provides a quantum theory of geometry with discrete eigenvalues of geometric operators such as area or volume, see $[3,4,5,6]$ and references therein. More importantly, for the first time it was possible to make mathematical sense out of the famous Wheeler-DeWitt-equation and construct a well defined Hilbert space. In the period from 1996 until today, the focus of research has shifted towards applications of the framework, most notably Loop Quantum Cosmology, the quantisation of cosmological models using LQG techniques [7], and the derivation of the Bekenstein-Hawking entropy from a microscopic analysis [8].

While todays measurements indicate the dimensionality of spacetime to be $3+1$ and supersymmetry has not been found yet, the ideas of extra dimensions and supersymmetry are still attracting a lot of interest, most notably in the context of String Theory, a mainly perturbative approach to quantum gravity which aims at providing the framework for a unified quantum theory of all forces $[9,10]$. Since the cosmological sector of String Theory has been addressed in the context of String cosmology [12] and also a microscopic derivation of the black hole entropy is available [13], it sounds natural to try to compare these two approaches to quantum gravity, starting with their results in these symmetry-reduced situations. Presently, the best one could do is to compare these results in $3+1$ spacetime dimensions by dimensionally reducing String Theory. The problem however, also known as the landscape, is that many choices have to be made in order to arrive at a $3+1$ dimensional reduction of String Theory and it is not clear which one should be preferred. In 10/11 dimensions, the fundamental dimensions of Superstring/ M-Theory, much less choices have to be made in order to describe the theories. Therefore, applying the techniques of LQG to higher dimensional Supergravities [14], which by symmetry arguments are expected to be the low energy effective field theory limits of Superstring/M-Theory, seems more favourable in order to build a bridge between these approaches to Quantum Gravity and we will follow this route in this letter. Despite its obvious applications, the literature on generalisations of LQG to higher dimensional Supergravities is rather scarce [15]. The reason for this can be traced back the very foundation of the LQG programme, namely the possibility of rewriting General Relativity in $3+1$ dimensions classically as an $\mathrm{SO}(3)$ gauge theory. From a Lagrangian point of view, the group $\mathrm{SO}(3)$ appears after gauge fixing the time-like part of the Lorentz group $\mathrm{SO}(1,3)$ in a vierbein formulation of General Relativity. It thus sounds plausible that the derivation can simply be repeated in any dimension, which however is not possible [16]. In order to understand the problem, it is more instructive to look at a Hamiltonian derivation of the connection formulation. Here, one starts from the ADM formulation of Hamiltonian General Relativity [17], in which a globally hyperbolic spacetime of dimension $D+1$ is foliated into $D$-dimensional constant time hypersurfaces with Euclidean signature endowed with a metric $q_{a b}$, where $a, b, . .=1, . ., D$ are spatial indices on the leaves of the spacetime foliation. The remaining information of the spacetime metric, more precisely the embedding information of the constant time hypersurfaces into the spacetime manifold, are encoded in the extrinsic curvature $K_{a b}$ which in turn enters the definition of $P^{a b}$, the momentum conjugate to $q_{a b}$. To obtain an $\mathrm{SO}(3)$ gauge symmetry in the $3+1$-dimensional case, an extended phase space is introduced, coordinatised by a co-dreibein defined by $q_{a b}=e_{a}^{i} e_{b}^{j} \delta_{i j}$ and its conjugate momentum $K_{a}^{i}=K_{a b} e^{b i}$, where $i, j, . .=1, . ., 3$ are so(3) indices in the fundamental representation. Finally, one adds $K_{a}^{i}$ to the spin connection $\Gamma_{a i j}$ annihilating the co-dreibein $e_{a}^{i}$ to form the Ashtekar-Barbero 
connection

$$
A_{a i j}=\Gamma_{a i j}+\gamma \varepsilon_{i j k} K_{a}^{k}
$$

where $\gamma \in \mathbb{R}$ is a free parameter called the Immirzi parameter. Since we are adding the extrinsic curvature, transforming in the fundamental representation of $\mathrm{SO}(3)$, to the spin connection, transforming in the adjoint representation, it becomes clear that this construction can only work for very special groups and a scan of the classical Lie groups reveals that $\mathrm{SO}(3)$ is singled out.

At the quantum level however, the restriction to a special dimension or gauge group is not necessary. In fact, the quantisation methods are formulated for any dimension and compact gauge group $\mathscr{G}$, the only restrictions being that:

I. The connection $A_{a}^{j}$ and its canonically conjugate momentum are real valued, where $a=$ $1, . ., D$ and $j=1, . . \operatorname{dim} \mathscr{G}$.

II. The Poisson algebra is of the usual, simple form

$$
\begin{aligned}
& \left\{A_{a}^{j}(x), A_{b}^{k}(y)\right\}=\left\{E_{j}^{a}(x), E_{k}^{b}(y)\right\}=0, \\
& \left\{E_{j}^{a}(x), A_{b}^{k}(y)\right\}=-\gamma \delta_{b}^{a} \delta_{j}^{k} \delta(x, y) .
\end{aligned}
$$

III. The gauge group $\mathscr{G}$ is compact.

It follows that if one is able to rewrite higher dimensional Supergravities as Yang-Mills theories with the above properties, one basically gets the quantisation thereof for free.

The intuition on how to construct a connection formulation with the above listed properties in higher dimensions can be obtained from the procedure of gauge unfixing [18]. By performing the canonical analysis of the $D+1$-dimensional Palatini formulation of General Relativity [19], on arrives at an $\mathrm{SO}(1, D)$ Yang-Mills theory phase space, however, it is subject to second class constraints, the simplicity constraint $S$ (which will be explained below) and a second class partner, which in particular implies that the connection is not self-commuting with respect to the corresponding Dirac bracket [20] and properties II. and III. listed above are therefore not satisfied. Instead of using the Dirac bracket or choosing time gauge, it was shown in [19] that the second class partner of the simplicity constraint can be removed without changing the physical content of the theory by the machinery of gauge unfixing. One is left with a connection formulation with only first class constraints and a Poisson-commuting connection. The drawback however is that property III. is still not satisfied because of the non-compact gauge group $\mathrm{SO}(1, D)$.

The Lagrangian derivation of this connection formulation now suggests the existence of a dual Hamiltonian derivation, i.e. an extension of the ADM phase space of General Relativity, the details of which can be deduced by performing the symplectic reduction with respect to the simplicity constraint [21] of the formulation obtained by gauge unfixing. The idea is to decompose the $\operatorname{SO}(1, D)$ connection $A_{a I J}$, with $I, J, . .=0, . . D$ being $\mathrm{SO}(1, D)$ Lie algebra indices, as

$$
A_{a I J}=\Gamma_{a I J}^{\mathrm{HYB}}+K_{a I J}
$$

where $\Gamma_{a I J}^{\mathrm{HYB}}$ [21], called the hybrid spin connection, is a generalisation of the usual spin connection $\Gamma_{a i j}$ to the case where the internal space is one dimension bigger than the spatial hypersurface. 
The boost part of $K_{a I J}$ can be shown to be related to the extrinsic curvature $K_{a b}$ while the remaining part turns out to be pure gauge. Starting from the ADM phase space, one can now reverse the process and rewrite the ADM variables, the spatial metric $q_{a b}$ and its conjugate momentum $P^{a b}\left(q_{c d}, K_{c d}\right)$ as functions of an $\mathrm{SO}(1, D)$ connection and its conjugate momentum $\pi^{a I J}$ inspired by (1) and show that the ADM Poisson brackets are reproduced [22]. The simplicity constraint $S$ enters the construction as a bridge between the proposed Yang-Mills version of General Relativity and the vielbein formulation. In fact, on the constraint surface $S=0, \pi^{a I J} \propto n^{[I} e^{a \mid J]}$, where $e^{a J}$ is the pullback of the spacetime vielbein to the spatial hypersurfaces and $n^{I}$ is the (up to sign) unique unit normal on $e^{a I}$, i.e. $n^{I} e_{I}^{a}=0$. The spatial metric $q_{a b}$ can thus be written as a function of $\pi^{a I J}$. In this sense, the connection $A_{a I J}$ is canonically conjugate to the spatial metric whose quantisation can be obtained, in simplified terms, by representing $\pi^{a I J}$ as a derivative operator [23].

As a next step, one realises that nothing in the calculations actually depends on whether the internal space is of Lorentzian or Euclidean signature, the reason of which can be traced back to the fact that the Hamiltonian formulations of Lorentzian and Euclidean General Relativity lead to the same phase space, while the signature of spacetime appears as a relative sign in the Hamiltonian constraint. Lorentzian General Relativity can thus be expressed as an $\mathrm{SO}(D+1)$ Yang-Mills theory, and a loop quantisation can be performed [23].

As compared to the $\mathrm{SO}(3)$ based standard LQG, the Hamiltonian constraint is modified by a new term, which can be interpreted as a gauge invariant extension of the original Hamiltonian with respect to the simplicity constraint which is an integral part of the new connection formulation. The gauge transformations generated by the simplicity constraint can be shown to change a certain part of the torsion of $A_{a I J}$. While this torsion symmetry and the switch of internal signature can be understood from a Hamiltonian point of view, it is unclear if they can be translated to a manifestly covariant Lagrangian framework. The simplicity constraint, which is already well known from spin foam models [24, 25], can be quantised and has to be treated at the quantum level. It restricts irreducible representations of $\mathrm{SO}(D+1)$ appearing in the quantum theory to be spherical [26] and furthermore imposes constraints on the intertwining maps between them, see [27] for first steps in the canonical theory.

The following remark is due at this point for the case of $D=3$ : In the usual formulation, the connection $A^{\mathrm{LQG}}$ is an $\mathrm{SO}(3)$ connection and is related to the extrinsic curvature by

$$
A_{a j k}^{\mathrm{LQG}}-\Gamma_{a j k}^{\mathrm{SPIN}}[e]=\gamma \varepsilon_{j k l} K_{a}^{l} ; A_{a 0 j}^{\mathrm{LQG}}=\Gamma_{a 0 j}^{\mathrm{SPIN}}[e] \equiv 0,
$$

where $K_{a b}=K_{a}^{j} e_{b}^{j}$ and where $\Gamma_{a j k}^{\mathrm{SPIN}}[e]$ is the spin connection of the co-dreibein $e_{a}^{j}$. Neither $A^{L Q G}$ nor $\Gamma^{\mathrm{SPIN}}$ have a "boost" part, the information about the extrinsic curvature is encoded in the rotational part of $A^{\mathrm{LQG}}$ and depends on the Immirzi parameter $\gamma$. On the other hand, in the "time gauge" $n^{I}=\delta_{0}^{I}$

$$
A_{a j k}^{\mathrm{NEW}}-\Gamma_{a j k}^{\mathrm{HYB}}[\pi] \approx \mathrm{S}-\text { gauge, } A_{a 0 j}^{\mathrm{NEW}}-\Gamma_{a 0 j}^{\mathrm{HYB}}[\pi]=\beta K_{a}^{j},
$$

where $\Gamma^{\mathrm{HYB}}[\pi]$ is the hybrid connection built from $\pi$ and $\beta \in \mathbb{R}$ is a free parameter. Therefore the information about the extrinsic curvature is encoded in the boost part of the new connection and depends on the parameter $\beta$. It transpires that the Immirzi parameter $\gamma$ and $\beta$ have nothing to do with each other and that the new formulation is a rather different extension of the ADM phase space as compared to the LQG formulation even in $D=3$ which nevertheless have the same symplectic 
reduction, namely the ADM phase space. In [19] we show that for $D=3$ one can generalise the exposition given so far by considering a 2 parameter family of connection formulations depending on both $\gamma, \beta$. The essential features remain the same, the connection remains Poisson commuting. Ultimately, for $D=3$, both, the quantisation using Ashtekar Barbero variables and the one employing the new variables proposed here, should at least yield the same semiclassical physics. Although not strictly forced on us, the stronger requirement that the joint kernel of the simplicity and $\mathrm{SO}(4) \mathrm{Gau} ß$ constraint in the quantum theory built from the new variables and the $\mathrm{SU}(2)$ invariant kinematical Hilbert space when using Ashtekar-Barbero variables are unitarily equivalent gives a tentative guideline for the implementation of the quantum simplicity constraints, and a similar requirement is usually posed in spin foams.

It should be noted that the new variables presented in this letter also provide a new starting point for comparing the canonical approach of LQG to the spin foam models which use a path integral formulation. More precisely, the quantum simplicity constraint is a cornerstone in the spin foam models, however it is solved classically in the usual LQG formulation and a relation between the two approaches could be established only on a kinematical level. In order to establish a dynamical relation, e.g. by showing that the spin foam path integral projects onto the kernel of the Hamiltonian constraint, it seems favourable to start from the canonical formulation presented in this letter since it is closer to the starting point of the spin foam quantisation, a constrained BF-theory. Also, the appearance of second class constraints (second class partners to the simplicity constraints) in the canonical analysis has been argued to be a problem for the spin foam quantisation [28]. On the one hand, we have shown that the second class partners can be removed by gauge unfixing and the simplicity constraint can be classically treated as a first class constraint, on the other hand, the price to pay was a new term in the Hamiltonian constraint which does not naturally appear in the canonical analysis of the action on which the spin foam models are based. Further research in this direction thus has to be conducted, however the new variables seem to be a good starting point to make progress with these important questions.

In order to be able to treat Supergravities, the new connection formulation has to be extended to matter fields. Scalar fields as well as gauge bosons can be treated in the usual manner [29]. Dirac Fermions however are a bit more involved, since they transform under the spinor representation of $\mathrm{SO}(1, D)$. During the canonical analysis of the corresponding action, one uses the time gauge and thus reduces the effective gauge symmetry to $\mathrm{SO}(D)$. The idea is now to act with $\mathrm{SO}(D+1)$ on the (complex) spinorial representation space of $\mathrm{SO}(1, D)$ and to rewrite the Hamiltonian constraint in an $\mathrm{SO}(D+1)$ invariant way such that it reduces to the $\mathrm{SO}(D)$ invariant version derived from the canonical analysis upon choosing the time gauge and solving the simplicity constraint [30]. The possibility of doing so heavily relies on the strong similarity of the representations of the Clifford algebras of $\mathrm{SO}(1, D)$ and $\mathrm{SO}(D+1)$, Dirac's $\gamma$-matrices, which differ only by a factor of $i$ in $\gamma_{0}$.

The situation is however more complicated as soon as one turns to Rarita-Schwinger fields, which obey a Majorana condition. In the interesting dimensions $D+1=4,10,11$, there exist real representations of the Lorentz group $\mathrm{SO}(1, D)$ and thus real fermions can be used, however, due to the above factor of $i$, the action $\mathrm{SO}(D+1)$ on spinor space is necessarily complex in those dimensions. The problem can be resolved by observing that the complex "directions" of $\mathrm{SO}(D+1)$ are along a real normal $N^{I}$, transforming in the fundamental (real) representation of $\mathrm{SO}(D+1)$. It is possible to write a compound object of this normal and the Rarita-Schwinger field on which the action of 
$\mathrm{SO}(D+1)$ can be defined in such a way that it respects the reality condition of the Majorana spinor [31]. In a precise sense, the normal field $N^{I}$ keeps track of the deviation of the components of the Rarita-Schwinger field from the real axis and compensates in the compound (physical) object for the complex (gauge) rotations away from the initial, real values. Consequently, $N^{I}$ has to be introduced as a new canonical variable and included in the quantisation procedure. Interestingly, the normal field can be interwoven in the gravitational degrees of freedom by using a linearised version of the simplicity constraint [31] already familiar from the new spin foam models [25].

On top of fields already discussed, Abelian p-form fields are a natural ingredient to higher dimensional Supergravities, most notably the 3-index photon of $11 d$ Supergravity and the 2-form KalbRamond field from the $10 d$ Supergravities. As an example, we studied the 3-index photon which is subject to a complication coming from the Chern-Simons term in the 11d Supergravity action [32]. More precisely, the gauge constraint is altered by a term which transforms the momentum conjugate to the 3-index photon in a non-trivial way. One can however perform a reduced phase space quantisation by using only gauge invariant quantities and find a Hilbert space representation of the Weyl elements by using a state of the Narnhofer-Thirring type [33]. Interestingly, due to the non-trivial gauge invariant functions, the Weyl algebra is twisted by a contribution proportional to the level of the Chern-Simons theory.

Thus, we believe to have provided the tools for an LQG type of quantisation of at least a subset of Supergravity theories in higher dimensions, notably $11 \mathrm{~d}$ Supergravity and $4 \mathrm{~d} N=8$ Supergravity which can be obtained from the $11 \mathrm{~d}$ theory by dimensional reduction as well as some Supergravity theories in $10 \mathrm{~d}$ and $4 \mathrm{~d}$. This by far does not exhaust all of them. Some Supergravity theories contain non Abelian p-form fields for whose quantisation most probably requires techniques from higher gauge theory [34]. Other Supergravity theories only have an on shell formulation, that is, the algebra of local supersymmetry constraints closes only modulo the equations of motion making them second class off shell. We do not have yet any concrete proposal for how to deal with these kinds of Supergravity Theories, possibly ideas from gauge unfixing may be applied in order to turn such a system first class.

Many questions are not answered by our work, for instance:

The precise relation with String Theory/M Theory is not provided. Our formulation works but is most certainly not the most elegant one. The treatment of the simplicity constraint remains unsatisfactory. A formulation in terms of superfields is maybe more desirable. We hope to be able to improve on this in future publications and have many other applications in mind such as revisiting the AdS/CFT correspondence [35] and its integrability structure [36] in the light of the proposed quantisation of Supergravity.

\section{Acknowledgments}

NB and AT thank the German National Merit Foundation for financial support and Matthias Blau for correspondence about Hamiltonian Supergravity. TT thanks Dieter Lüst and Hermann Nicolai for motivational discussions about higher dimensional Ashtekar variables and their application to Supergravity and String Theory. We want to thank the referee for valuable comments. 


\section{References}

[1] N. Arkani-Hamed, F. Cachazo and J. Kaplan, What is the Simplest Quantum Field Theory?, JHEP 1009 (2010) 016 [arXiv:0808.1446 [hep-th] ], Kallosh R., On UV Finiteness of the Four Loop N=8 Supergravity, JHEP 0909 (2009) 116 [arXiv:0906.3495 [hep-th] ].

[2] A. Ashtekar, New Hamiltonian formulation of general relativity, Phys. Rev. D36 (1987) 1587-1602.

[3] C. Rovelli, Quantum Gravity, Cambridge University Press, Cambridge 2004.

[4] T. Thiemann, Modern Canonical Quantum General Relativity, Cambridge University Press, Cambridge 2007.

[5] M. Bojowald, Canonical Gravity and Applications: Cosmology, Black Holes, and Quantum Gravity, Cambridge University Press, Cambridge 2011.

[6] R. Gambini and J. Pullin, A First Course in Loop Quantum Gravity, Oxford University Press, Oxford 2011.

[7] M. Bojowald, Loop Quantum Cosmology, Living Rev. Relativity 11, (2008), 4. URL (cited on July 6, 2011): http://www.livingreviews.org/lrr-2008-4

[8] I. Agullo, J. Fernando Barbero, E. F. Borja, J. Diaz-Polo and E. J. S. Villasenor, Detailed black hole state counting in loop quantum gravity, Phys. Rev. D82 (2010) 084029 [arXiv: 1101.3660 $[\mathrm{gr}-\mathrm{qC}]]$.

[9] M. B. Green, J. Schwarz and E. Witten, Superstring Theory, vol. 1,2, Cambridge University Press, Cambridge 1987.

[10] J. Polchinski, String Theory, vol.1,2, Cambridge University Press, Cambridge 1998.

[11] L. McAllister and E. Silverstein, String cosmology: a review, Gen. Rel. Grav. 40 (2008) 565-605.

[12] J. Erdmenger, String Cosmology: Modern String Theory Concepts from the Big Bang to Cosmic Structure, Wiley-VCH, 2009.

[13] A. Strominger and C. Vafa, Microscopic origin of the Bekenstein-Hawking entropy, Phys. Lett. B379 (1996) 99-104 [arXiv: hep-th/9601029v2], J. M. Maldacena, Black holes in string theory, arXiv:hep-th/9607235.

[14] P. van Nieuwenhuizen, Supergravity, Phys. Rep. 68 (1981) 189.

[15] S. Melosch and H. Nicolai, New canonical variables for D $=11$ supergravity, Phys. Lett. B416 (1998) 91-100 [arXiv: hep-th/9709227].

[16] M. Han, Y. Ma, Y. Ding and L. Qin, Hamiltonian analysis of n-dimensional Palatini gravity with matter, Mod. Phys. Lett. A20 (2005) 725-732 [arXiv:gr-qc/ 0503024 ].

[17] R. Arnowitt, S. Deser and C. W. Misner, The dynamics of general relativity, in Gravitation: An introduction to current research, L. Witten (ed.), New York, Wiley 1962.

[18] P. Mitra and R. Rajaraman, Gauge Invariant Reformulation Of An Anomalous Gauge Theory, Phys. Lett. B225 (1989) 267-271, R. Anishetty and A. S. Vytheeswaran, Gauge invariance in second-class constrained systems, Journal of Physics A26 (1993) no. 20 5613Đ5619, A. S. Vytheeswaran, Gauge unfixing in second class constrained systems, Annals Phys. 236 (1994) 297-324.

[19] N. Bodendorfer, T. Thiemann and A. Thurn, New Variables for Classical and Quantum Gravity in all Dimensions II. Lagrangian Analysis, arXiv:1105.3704 [gr-qC] . 
[20] S. Alexandrov, $S O(4, C)$ covariant Ashtekar-Barbero gravity and the Immirzi parameter, Class. Quant. Grav. 17 (2000) 4255-4268 [arXiv: gr-qc/ 0005085 ].

[21] P. Peldan, Actions for gravity, with generalisations: a review, Class. Quant. Grav. 11 (1994) 1087-1132 [arXiv: gr-qc/9305011].

[22] N. Bodendorfer, T. Thiemann and A. Thurn, New Variables for Classical and Quantum Gravity in all Dimensions I. Hamiltonian Analysis, arXiv:1105.3703 [gr-qC] .

[23] N. Bodendorfer, T. Thiemann and A. Thurn, New Variables for Classical and Quantum Gravity in all Dimensions III. Quantum Theory, arXiv:1105.3705 [gr-qc] .

[24] J. W. Barrett and L. Crane, Relativistic spin networks and quantum gravity, J. Math. Phys. 39 (1998) 3296-3302 [arXiv: gr-qc/9709028v2].

[25] J. Engle, R. Pereira and C. Rovelli, The Loop-quantum-gravity vertex-amplitude, Phys. Rev. Lett. 99 (2007) 161301 [arXiv: 0705.2388 [ gr-qc] ], L. Freidel and K. Krasnov, A New Spin Foam Model for 4d Gravity, Class. Quant. Grav. 25 (2008) 125018 [arXiv:0 0708.1595 [gr-qc]].

[26] L. Freidel, K. Krasnov and R. Puzio, BF description of higher dimensional gravity theories, Adv. Theor. Math. Phys. 3 (1999) 1289-1324 [arXiv: hep-th/9901069].

[27] N. Bodendorfer, T. Thiemann and A. Thurn, On the Implementation of the Canonical Quantum Simplicity Constraint, arXiv:1105.3708 [gr-qc] .

[28] S. Alexandrov and P. Roche, Critical Overview of Loops and Foams, Phys. Rept. 506 (2011) 41-86 [arXiv:1009.4475v1 [gr-qc]].

[29] T. Thiemann, Quantum spin dynamics (QSD): V. Quantum gravity as the natural regulator of the Hamiltonian constraint of matter quantum field theories, Class. Quant. Grav. 15 (1998) 1281-1314 [arXiv: gr-qc/9705019], T. Thiemann, Kinematical Hilbert spaces for fermionic and Higgs quantum field theories, Class. Quant. Grav. 15 (1998) 1487-1512 [arXiv: gr-qc/ 9705021 ].

[30] N. Bodendorfer, T. Thiemann and A. Thurn, New Variables for Classical and Quantum Gravity in all Dimensions IV. Matter Coupling, arXiv:1105.3706 [gr-qc] .

[31] N. Bodendorfer, T. Thiemann and A. Thurn, Towards Loop Quantum Supergravity (LQSG) I. Rarita-Schwinger Sector, arXiv:1105.3709 [gr-qc] .

[32] N. Bodendorfer, T. Thiemann and A. Thurn, Towards Loop Quantum Supergravity (LQSG) II. p-Form Sector, arXiv:1105.3710 [gr-qC].

[33] H. Narnhofer and W. Thirring, Covariant QED without indefinite metric, Rev. Math. Phys. 4 (1992) 197-211.

[34] J. C. Baez and J. Huerta, An Invitation to Higher Gauge Theory, Gen. Rel. Grav. 43 (2011) 2335-2392 [arXiv:1003.4485 [hep-th]].

[35] J. L. Petersen, Introduction to the Maldacena conjecture on AdS / CFT, Int. J. Mod. Phys. A14 (1999) 3597-3672 [arXiv:hep-th/9902131].

[36] N. Beisert et al., Review of AdS/CFT Integrability: An Overview, Lett. Math. Phys. 99 (2012) 3-32 [arXiv:1012.3982 [hep-th]]. 